\title{
Role of chemical substitution in the photoluminescence properties of cerium samarium tungstates $\mathrm{Ce}_{(2-\mathrm{x})} \mathrm{Sm}_{\mathrm{x}}\left(\mathrm{WO}_{4}\right)_{3}(0 \leq \mathrm{x} \leq 0.3)$
}

\author{
$\underline{\text { K. Derraji }}{ }^{1}$, C. Favotto ${ }^{1}$, J-C, Valmalette ${ }^{1}$, S. Villain ${ }^{1}$, J-R. Gavarri ${ }^{1}$, G. Nolibe ${ }^{2}$, \\ A. Lyoussi ${ }^{3}$ and F. Guinneton ${ }^{1 *}$ \\ ${ }^{1}$ Université de Toulon, Aix-Marseille Univ., CNRS, IM2NP, France \\ ${ }^{2}$ CeSigma S.A, Le Pradet, France \\ ${ }^{3}$ CEA Cadarache, St Paul lez Durance, France \\ *contact: guinneton@univ-tln.fr
}

\begin{abstract}
.
In the general framework of the development of materials with tunable photoluminescence, a series of cerium samarium tungstates $\mathrm{Ce}_{(2-\mathrm{x})} \mathrm{Sm}_{\mathrm{x}}\left(\mathrm{WO}_{4}\right)_{3}$ with $\mathrm{x} \leq 0.3$ was synthesized by a coprecipitation method followed by thermal treatment at $1000{ }^{\circ} \mathrm{C}$. The polycrystalline compounds were characterized by X-ray diffraction, scanning electron microscopy and photoluminescence experiments. In the present work, the objective would be to determine the role of PL emitting centers in the variations of PL intensities. Firstly, Rietveld analysis showed a decrease of cell parameters and confirmed that a solid solution was obtained. Diffraction profile analyses showed that structural distortions increasing with composition $\mathrm{x}$ were observed: they were ascribed to difference in cation sizes of $\mathrm{Ce}^{3+}$ and $\mathrm{Sm}^{3+}$, and to defects generated during crystal growth. The photoluminescence (PL) spectra were obtained under X-Ray (45 kV-35 mA) and UV (364.5 nm) excitations. Two PL emissions of $\mathrm{Ce}^{3+}$ were observed only under UV excitation. Four PL emissions of $\mathrm{Sm}^{3+}$ were observed under UV and X-ray excitations, and their intensities decreased with increasing composition $\mathrm{x}$. Two additional transitions were observed under UV and X-ray excitations: they were attributed to oxygen vacancy defects. In the range 800 to $1000 \mathrm{~nm}$, an increasing IR emission is observed: it was

ascribed to emissions due to other oxygen vacancies. The main results are reported in Table 1. The chromaticity diagram (see Figure 1) showed that the colors associated with PL responses vary with Sm composition and excitation energies. This offers the opportunity to develop materials with tunable PL. To better understand this complex behavior, now, we plan to study the solid solution in the composition range $\mathrm{x}>0.3$.
\end{abstract}


Table 1: Photoluminescence emissions under UV and X-ray excitations observed in the series $\mathrm{Ce}_{2-\mathrm{x}} \mathrm{Sm}_{\mathrm{x}}\left(\mathrm{WO}_{4}\right)_{3}$. Transitions generated by presence of oxygen vacancies $\mathrm{Vx}, \mathrm{V}^{\circ}$ and $\mathrm{V}^{\circ \circ}$.

\begin{tabular}{|c|c|c|c|c|}
\hline \multicolumn{2}{|c|}{ UV excitation } & \multicolumn{2}{|c|}{ X-ray excitation } & \multirow{2}{*}{$\begin{array}{c}\text { Attributions } \\
\text { Transitions } \mathrm{Ce}^{3+} \text { and } \mathrm{Sm}^{3+}\end{array}$} \\
\hline $\begin{array}{c}\text { Wavelength } \\
\text { (nm) } \\
\text { Color }\end{array}$ & Energy $(\mathrm{eV})$ & $\begin{array}{l}\text { Wavelength }(\mathrm{nm}) \\
\text { Color }\end{array}$ & Energy $(\mathrm{eV})$ & \\
\hline $\begin{array}{c}497.75 \\
\text { Green } \\
\end{array}$ & 2.49 & No emission & & Ce: $\quad{ }^{2} \mathrm{~T} 2 \mathrm{~g} /{ }^{2} \mathrm{D}_{3 / 2} \rightarrow{ }^{2} \mathrm{~F}_{7 / 2}$ \\
\hline $\begin{array}{l}524.61 \\
\text { Green }\end{array}$ & 2.36 & No emission & & Ce: $\quad{ }^{2} \mathrm{~T} 2 \mathrm{~g} /{ }^{2} \mathrm{D}_{5 / 2} \rightarrow{ }^{2} \mathrm{~F}_{5 / 2}$ \\
\hline $\begin{array}{l}564.33 \\
\text { Yellow } \\
\end{array}$ & 2.19 & $\begin{array}{l}563 \\
\text { Yellow } \\
\end{array}$ & 2.20 & ${ }^{4} \mathrm{G}_{5 / 2}->{ }^{6} \mathrm{H}_{5 / 2}$ \\
\hline $\begin{array}{l}600.50 \\
\text { Orange }\end{array}$ & 2.064 & $\begin{array}{c}599 \\
\text { Orange }\end{array}$ & 2.070 & Sm: $\quad{ }^{4} \mathrm{G}_{5 / 2}->{ }^{6} \mathrm{H}_{7 / 2}$ \\
\hline $\begin{array}{l}616.56 \\
\text { Orange }\end{array}$ & 2.013 & $\begin{array}{c}617 \\
\text { Orange }\end{array}$ & 2.009 & $\mathrm{~V}^{\circ *} \rightarrow \mathrm{V}^{\circ}$ \\
\hline $\begin{array}{c}643.2 \\
\text { Red }\end{array}$ & 1.927 & $\begin{array}{l}644 \\
\text { Red }\end{array}$ & 1.925 & Sm: $\quad{ }^{4} \mathrm{G}_{5 / 2}->{ }^{6} \mathrm{H}_{9 / 2}$ \\
\hline $\begin{array}{l}667 \\
\text { Red }\end{array}$ & 1.859 & $\begin{array}{l}669 \\
\text { Red }\end{array}$ & 1.853 & $\mathrm{~V}^{\mathrm{x} *} \rightarrow \mathrm{V}^{\mathrm{x}}$ \\
\hline $\begin{array}{c}705 \\
\text { Dark red }\end{array}$ & 1.759 & $\begin{array}{c}705 \\
\text { Dark red }\end{array}$ & 1.759 & Sm: $\quad{ }^{4} \mathrm{G}_{5 / 2} \rightarrow{ }^{6} \mathrm{H}_{11 / 2}$ \\
\hline $\begin{array}{c}900 \\
\text { Large IR }\end{array}$ & 1.38 & $\begin{array}{c}880 \\
\text { Large IR }\end{array}$ & 1.41 & $\mathrm{~V}^{\circ} \rightarrow$ holes $\mathrm{O} 2 \mathrm{p}$ \\
\hline $\begin{array}{c}950 \\
\text { Large IR }\end{array}$ & 1.30 & $\begin{array}{c}930 \\
\text { Large IR }\end{array}$ & 1.33 & $\mathrm{~V}^{\mathrm{x}} \rightarrow$ holes $\mathrm{O} 2 \mathrm{p}$ \\
\hline
\end{tabular}

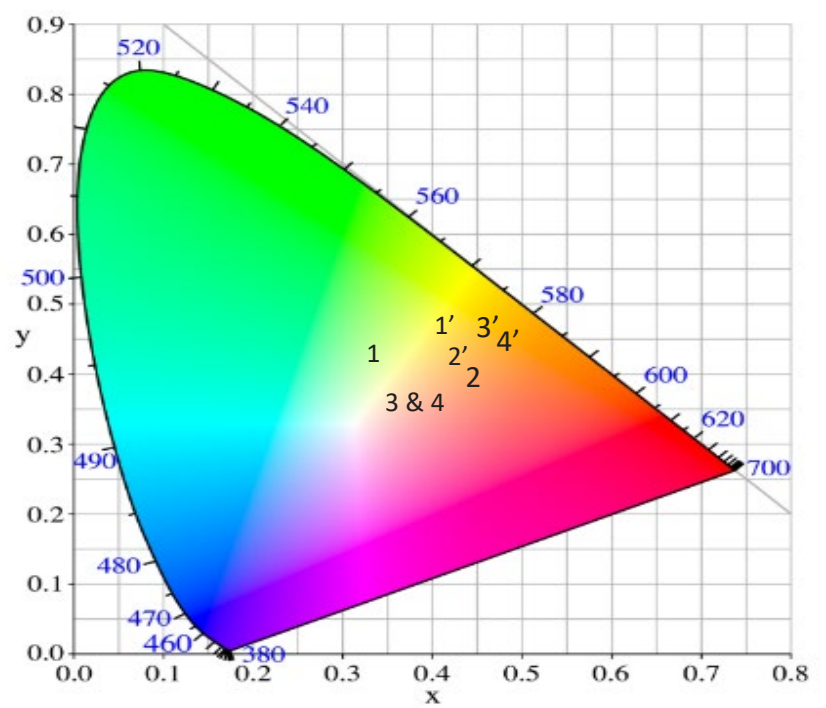

Figure 1: Chromaticity diagram for $\mathrm{Ce}_{2-\mathrm{x}} \mathrm{Sm}_{\mathrm{x}}\left(\mathrm{WO}_{4}\right)_{3}$ system under both excitations.

Under X-ray excitation, points are noted as 1 for $\mathrm{x}=0, \mathbf{2}$ for $\mathrm{x}=0.1, \mathbf{3}$ for $\mathrm{x}=0.2$ and $\mathbf{4}$ for $\mathrm{x}=0.3$. Under UV excitation points are noted as 1' for $\mathrm{x}=0, \mathbf{2}^{\prime}$ for $\mathrm{x}=0.1, \mathbf{3}^{\prime}$ for $\mathrm{x}=0.2, \mathbf{4}^{\prime}$ for $\mathrm{x}=0.3$.

Key words: samarium cerium tungstates, chemical substitution, structural distortions, photoluminescence. 